\title{
The differentiation potential of gingival mesenchymal stem cells induced by apical tooth germ cell-conditioned medium
}

\author{
YAN CHEN and HONGWEI LIU

\begin{abstract}
Department of Periodontology, Laboratory of Oral Biomedical Science and Translational Medicine,
\end{abstract} \\ School of Stomatology, Tongji University, Shanghai 200072, P.R. China
}

Received August 3, 2015; Accepted August 8, 2016

DOI: $10.3892 / \mathrm{mmr} .2016 .5726$

\begin{abstract}
Gingival-derived mesenchymal stem cells (GMSCs) have recently been harvested; however, the use of GMSCs in periodontal tissue engineering requires further study. The present study established an indirect co-culture system between rat apical tooth germ-conditioned medium (APTG-CM) and GMSCs, in order to determine the effects on periodontal tissue differentiation in vitro and in vivo. Using the limiting dilution technique, single-colony derived human GMSCs and periodontal ligament stem cells (PDLSCs) were isolated and expanded to obtain homogeneous populations. PDLSCs were used as a positive control group. Cell cycle distribution, alkaline phosphatase (ALP) activity, mineralization behavior, expression of genes associated with a cementoblast phenotype (osteocalcin, bone sialoprotein, ALP, type I collagen, cementum-derived protein 23), and in vivo differentiation capacities of GMSCs/PDLSCs co-cultured with APTG-CM were evaluated. Flow cytometry indicated that GMSCs and PDLSCs were positive for STRO-1 and CD105, whereas CD45 expression was negative. The cell types were capable of forming colonies, and of osteogenic and adipogenic differentiation in response to appropriate stimuli. The induced GMSCs and PDLSCs exhibited numerous characteristics associated with cementoblast lineages, as indicated by increased proliferation and ALP activity, and upregulated expression of cementum-associated genes in vitro. In vivo, cementum/periodontal ligament-like structures were shown to form along the dentin surface and ceramic bovine bone in GMSCs and PDLSCs induced by APTG-CM group. Conversely, vertical fibers could not insert in the control group, which was not co-cultured with APTG-CM. In conclusion, GMSCs are likely to have a role in periodontal tissue regeneration. In addition,
\end{abstract}

Correspondence to: Dr Hongwei Liu, Department of Periodontology, Laboratory of Oral Biomedical Science and Translational Medicine, School of Stomatology, Tongji University, 399 Middle Yanchang Road, Shanghai 200072, P.R. China

E-mail: hwliu@tongji.edu.cn

Key words: gingival-derived mesenchymal stem cells, periodontal ligament stem cells, cell differentiation, periodontal regeneration
APTG-CM was able to provide a cementogenic microenvironment and promote differentiation of GMSCs along the cementoblastic lineage.

\section{Introduction}

Periodontitis is a common inflammatory disease, which induces destruction of the tooth-supporting apparatus and may lead to tooth loss. However, current or emerging treatment options for periodontitis have limited and variable outcomes, or have yet to be developed for clinical use (1). The use of tissue engineering may be a possible therapeutic strategy for periodontal regeneration (2).

Periodontal ligament stem cells (PDLSCs) are effective and readily available cells for periodontal regeneration (3). However, due to the limitations in acquiring these cells, their application in clinical therapy is limited. The gingiva, which is an important component of periodontal tissue, exhibits promising regenerative ability and is able to rapidly repair injury. In addition, almost no scars remain after healing (4), it is available in large quantities and is easy to acquire. Previous studies have identified the presence of mesenchymal stem cells (MSCs) in human gingival connective tissue, and have reported on their morphological and functional characteristics (4-6). Therefore, gingival-derived MSCs (GMSCs) have recently received increased attention $(5,7,8)$.

Cell sheet technology refers to a scaffold-free tissue reconstruction technique. Based on a unique temperature responsive culture dish, cultured cells can be non-invasively harvested as intact contiguous sheets along with their deposited extracellular matrix $(9,10)$. Cell sheets may be used to avoid unforeseeable complications, including biomaterial degradation, inflammation and limited cell-seeding efficiency $(9,10)$. It has the natural reaggregation potential of monodispersed cells and the advantage of an affluent natural matrix (11-13).

It is well-known that tooth generation depends on the interaction between dental epithelium and MSCs (14); this interaction is also important in root and periodontal tissue formation (15-17). Therefore, it has being suggested that the interaction between dental epithelium and MSCs may be important in periodontal regeneration. During tooth development, the periodontium is formed by the apical mesenchyme of the tooth germ, which is associated with the expression of several signaling molecules, including transforming growth 
factor- $\beta$, Notch-1, fibroblast growth factors or bone morphogenetic proteins (18-22).

The present study used apical tooth germ-conditioned medium (APTG-CM) to induce differentiation of GMSCs and PDLSCs; subsequently, transplant complexes containing dentin slices, ceramic bovine bone $(\mathrm{CBB})$, and GMSC and PDLSC sheets were generated. The complex was subcutaneously transplanted into athymic mice for 8 weeks to observe periodontal regeneration.

\section{Materials and methods}

Isolation and characterization of PDLSCs and GMSCs. Gingival connective tissue samples were obtained from 10 healthy male donors (age, 15-25 years) at the Stomatology Hospital Affiliated to Tongji University (Shanghai, China). Under local anesthetic a 2x2x1 mm sample of keratinized gingival tissue was obtained from the maxillary tuberosity. In addition, human periodontal ligaments were obtained from healthy premolars extracted from 10 male donors (age, 13-25 years) undergoing orthodontic treatment at the Stomatology Hospital Affiliated to Tongji University (Shanghai, China). Sample collection conformed to a protocol approved by the ethical authorities at the School of Stomatology, Tongji University (Shanghai, China) and all donors provided informed consent.

Human GMSCs and PDLSCs were isolated as previously described $(3,6)$. Using the limiting dilution technique, single-colony derived human GMSCs and PDLSCs were isolated and expanded to obtain homogeneous populations. The cells were confirmed by fluorescence-activated cell sorting analysis using antibodies against CD45 (cat. no. FAB1430A), CD105 (cat. no. FAB10971P) and STRO-1 (cat. no. MAB1038; all obtained from R\&D Systems, Minneapolis, MN, USA), according to the manufacturer's protocol. The two types of stem cell were stimulated with osteogenic conditioned medium [100 nM dexamethasone, $5 \mathrm{mM} \beta$-glycerophosphate, $50 \mathrm{mg} / \mathrm{ml}$ ascorbate phosphate and $5 \%$ fetal bovine serum (FBS; Gibco; Thermo Fisher Scientific, Inc., Waltham, MA, USA)] and adipogenic conditioned medium [1 $\mathrm{mM}$ dexamethasone, $1 \mathrm{mg} / \mathrm{ml}$ insulin, $0.5 \mathrm{mM}$ 3-isobutyl-1-methylxanthine (Sigma-Aldrich; Merck Millipore, Darmstadt, Germany) and $5 \%$ FBS]. The conditions used for the induction of calcium accumulation and adipogenesis were the same as previously reported (12). Normal medium was used in the blank control group. Calcium accumulation was detected using 2\% Alizarin Red ( $\mathrm{pH}$ 4.2) staining (Sigma-Aldrich; Merck Millipore). Adipogenic cultures were stained with fresh Oil Red O solution (Sigma-Aldrich; Merck Millipore). Staining was observed by light microscopy (DX51; Olympus Corporation, Tokyo, Japan).

APTG-CM and co-culture system. All experiments involving the use of animals were reviewed and approved by the Animal Care Committee of Tongji University. Five-day postnatal Sprague-Dawley rats $(n=30$; male and female; weight, $10 \pm 2 \mathrm{~g}$; Shanghai SLAC Laboratory Animal Co., Ltd., Shanghai, China) were bred in plastic cages (5 mice/cage) under standard laboratory conditions with a $12 \mathrm{~h} \mathrm{light/dark} \mathrm{cycle,} \mathrm{at} 20^{\circ} \mathrm{C}$ with $48 \%$ humidity. Food and water were provided. The rats were sacrificed by decapitation under isoflurane anesthesia. Developing mandibular first molar tooth germs were then dissected from the jaws. The apical portion of the tooth germ was separated as previously reported (14), and was cultured in alpha-minimum essential medium (alpha-MEM; Gibco; Thermo Fisher Scientific, Inc.) supplemented with 10\% FBS. The primary APTG culture medium was changed every $48 \mathrm{~h}$ until $100 \%$ confluence for supernatant collection was reached. The cells were then centrifuged at $2,000 \times \mathrm{g}$ for $15 \mathrm{~min}$ at $4^{\circ} \mathrm{C}$. The supernatants were mixed with an equal volume of fresh alpha-MEM supplemented with $10 \%$ FBS and $50 \mu \mathrm{g} / \mathrm{ml}$ ascorbic phosphate (Sigma-Aldrich; Merck Millipore), which was used as APTG-CM for PDLSCs and GMSCs co-culture. Alpha-MEM supplemented with $10 \%$ FBS and $50 \mu \mathrm{g} / \mathrm{ml}$ ascorbic phosphate was used as a control medium.

Cell cycle analysis. Following 10 days of co-culture at $37^{\circ} \mathrm{C}$ with APTG-CM, single-cell suspensions of GMSCs and PDLSCs were harvested and were washed twice with phosphate-buffered saline (PBS). Subsequently, the cells were fixed in cold $70 \%$ dehydrated alcohol at $4^{\circ} \mathrm{C}$ for $24 \mathrm{~h}$. After further washing, cell suspensions were treated with RNase A and then stained with propidium iodide (PI; Sigma-Aldrich; Merck Millipore) at $4^{\circ} \mathrm{C}$ for $30 \mathrm{~min}$. The content of DNA was assayed by flow cytometry to confirm the cell cycle analysis and subjected to cell cycle analysis using MultiCycle software (Beckman Coulter, Inc., Brea, CA, USA. One million cells were counted per sample.

Alkaline phosphatase (ALP) activity assay. An ALP activity assay was performed to determine the influence of APTG-CM co-culture. Single-cell suspensions of GMSCs and PDLSCs were seeded at a density of $1 \times 10^{3}$ cells/well in 96-well plates. After 3, 6, 9, 12 and 15 days of co-culture with APTG-CM, the ALP activity of GMSCs and PDLSCs was detected using an ALP assay kit (Nanjing Jiancheng Bioengineering Institute, Nanjing, China.). The results were measured at $405 \mathrm{~nm}$ in a spectrophotometer using a multiplate reader (BioTek Instruments, Inc., Winooski, VT, USA).

\section{Reverse transcription-quantitative polymerase chain reaction} (RT-qPCR) analysis. The co-cultured GMSCs and PDLSCs, and the control cells, were harvested and RNA was extracted using TRIzol ${ }^{\circledR}$ reagent (Invitrogen; Thermo Fisher Scientific, Inc.) according to the manufacturer's protocol. The total RNA concentration was assessed using a NanoDrop 2000 spectrophotometer (Thermo Fisher Scientific, Inc.). RNA (1.0 mg) was reversed transcribed into cDNA using PrimeScript First Strand cDNA Synthesis kit (Takara Biotechnology Co., Ltd., Dalian, China) according to the manufacturer's protocols. Subsequently cDNA synthesis was conducted using a Superscript II First-strand cDNA synthesis kit (Invitrogen; Thermo Fisher Scientific, Inc.), and RT-qPCR was performed using a MyiQ2 Real-Time PCR Detection system (Bio-Rad Laboratories, Inc., Hercules, CA, USA). The thermocycling conditions were as follows: $95^{\circ} \mathrm{C}$ for $5 \mathrm{~min} ; 30$ cycles of $95^{\circ} \mathrm{C}$ for $30 \mathrm{sec}, 56^{\circ} \mathrm{C}$ for $30 \mathrm{sec}$ and extension at $72^{\circ} \mathrm{C}$ for $1 \mathrm{~min}$. Relative quantities of mRNA were calculated using the $2^{-\Delta \Delta \mathrm{Cq}}$ method (23) and normalized to housekeeping gene $\beta$-actin. The experiment was repeated $\geq 3$ times. Sequences for the sense 
Table I. Oligonucleotide primer sequences used in polymerase chain reaction.

\begin{tabular}{|c|c|c|}
\hline Gene & Primer & Sequence \\
\hline Osteocalcin & $\begin{array}{l}\text { Forward } \\
\text { Reverse }\end{array}$ & $\begin{array}{l}\text { 5'-GGGTCAGGAGGAGAATCGT-3' } \\
\text { 5'-GTGTCTTAGCAGGCAGGGA-3' }\end{array}$ \\
\hline Bone sialoprotein & $\begin{array}{l}\text { Forward } \\
\text { Reverse }\end{array}$ & $\begin{array}{l}\text { 5'-CTGCTTCCTCACTCCAGGAC-3' } \\
\text { 5'-ATTGAGAAAGCACAGGCCAT-3' }\end{array}$ \\
\hline Alkaline phosphatase & $\begin{array}{l}\text { Forward } \\
\text { Reverse }\end{array}$ & $\begin{array}{l}\text { 5'-GCCAAGAAAGCAGGAAAGTC-3' } \\
\text { 5'-GAGTACCAGTTGCGGTTCAC-3' }\end{array}$ \\
\hline Type I collagen & $\begin{array}{l}\text { Forward } \\
\text { Reverse }\end{array}$ & $\begin{array}{l}\text { 5'-CTGACCTTCCTGCGCCTGATGTCC-3' } \\
\text { 5'-GTCTGGGGCACCAACGTCCAAGGG-3' }\end{array}$ \\
\hline Cementum-derived protein 23 & $\begin{array}{l}\text { Forward } \\
\text { Reverse }\end{array}$ & $\begin{array}{l}\text { 5'-ATGGGCACATCAAGCACTGA-3' } \\
\text { 5'-CCCCATTAGTGTCATCCTGC-3' }\end{array}$ \\
\hline$\beta$-actin & $\begin{array}{l}\text { Forward } \\
\text { Reverse }\end{array}$ & $\begin{array}{l}\text { 5'-CAGGCTGTGCTATCCCTGTA-3' } \\
\text { 5'-CATACCCCTCGTAGATGGGC-3' }\end{array}$ \\
\hline
\end{tabular}

A

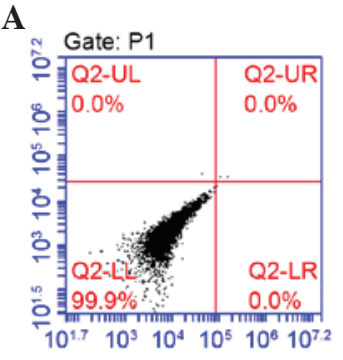

$\mathbf{E}$

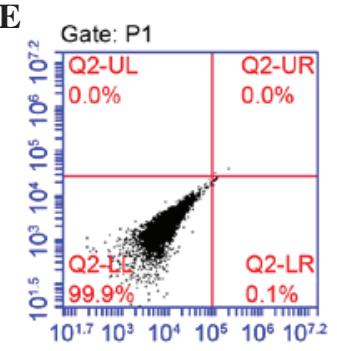

B

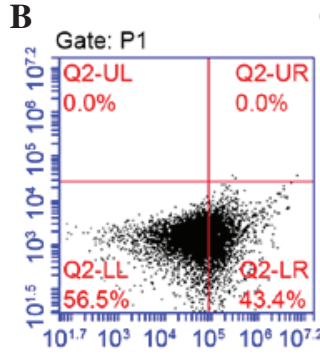

F

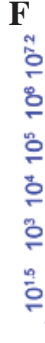

C

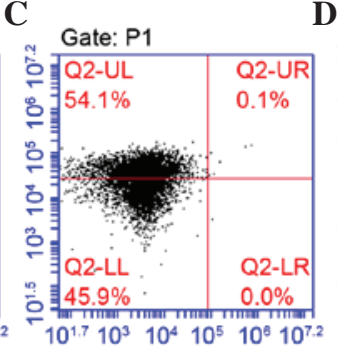

D

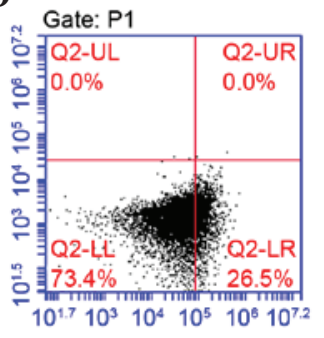

H

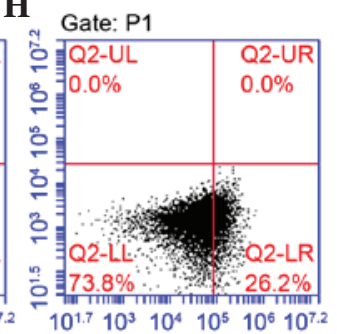

Figure 1. Flow cytometric analysis of gingival-derived mesenchymal stem cells (GMSCs) and periodontal ligament stem cells (PDLSCs). (A) Blank. (B) STRO-1, (C) CD105 and (D) CD45 markers identified in GMSCs. (E) Blank. (F) STRO-1, (G) CD105 and (H) CD45 markers identified in PDLSCs. STRO-1 and CD105 are surface epitopes of mesenchymal cells, and were positively expressed. CD45 is a surface epitope of hematopoietic stem cells, and was negatively expressed.

and anti-sense primers (Sangon Biotech Co., Ltd., Shanghai, China) used in the present study are listed in Table I.

GMSC and PDLSC sheets for the regeneration of cementum/periodontal ligament (PDL)-like complexes in mice. Single colony-derived GMSCs and PDLSCs wee cultured for 2 weeks to a density to form cell sheets, which were then detached from the bottom of the culture plates using a cell scraper. Cell sheets were used to wrap dentin slices (from root of tooth; $2 \times 3 \times 0.5 \mathrm{~mm}$ ) and CBB (Research and Development Center for Tissue Engineering, Fourth Military Medical University, Xi'an, China) was placed on either side. Surgical sutures were used to fasten the complex, and transplanted into the subcutaneous tissue of the upper backs of six-week-old immunodeficient mice $(n=30$; weight, $20 \pm 2 \mathrm{~g}$; Shanghai SLAC Laboratory Animal Co., Ltd.). A total of 30 complexes (10x3 groups) were implanted. Each immunodeficient mouse received one of three different complexes (APTG-CM GMSC sheet + dentin + CBB; GMSC sheet + dentin + CBB; and APTG-CM PDLSC sheet + dentin + CBB). The implants were recovered 8 weeks post-transplantation following decapitation under isoflurane anesthesia, were fixed in $4 \%$ paraformaldehyde for 2 days and were then decalcified for a further 28 days in 10\% EDTA ( $\mathrm{pH} 8.0$ ), prior to embedding in paraffin. For histological analysis, $5-\mu \mathrm{m}$ implant sections were prepared and stained with hematoxylin and eosin (H\&E; Sigma-Aldrich; Merck Millipore), according to the manufacturer's protocol (24). Staining was evaluated by light microscopy (DX51; Olympus Corporation).

Statistical analyses. Data are presented as the mean \pm standard deviation. Experiments were repeated three times. Statistical significance was analyzed using SPSS 17.0 software (SPSS, Inc., Chicago, IL, USA). One-way analysis of variance followed by Tukey's test was used to determine the significant differences among the groups. $\mathrm{P}<0.05$ was considered to 

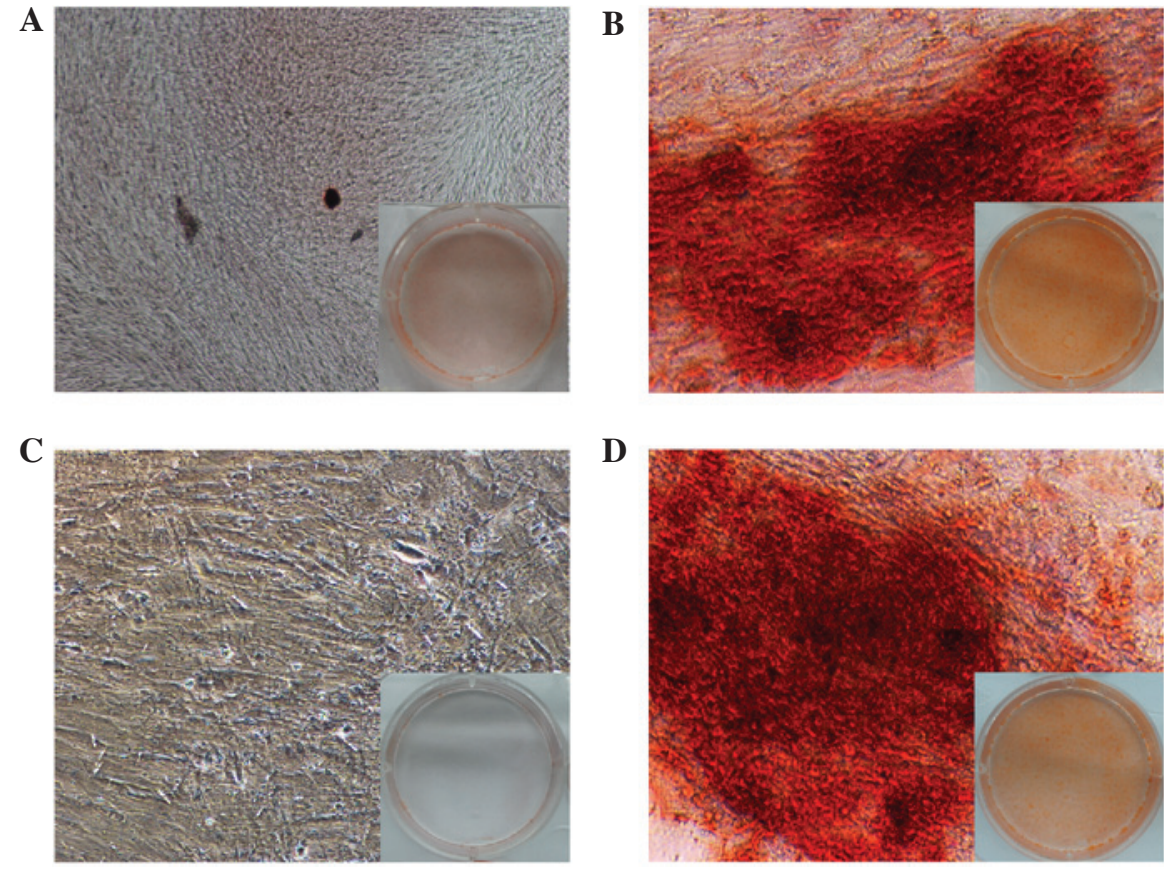

Figure 2. Osteogenic differentiation of (A and B) gingival-derived mesenchymal stem cells (GMSCs), and (C and D) periodontal ligament stem cells (PDLSCs). (A and C) Control groups; magnification, x40. (B and D) GMSCs and PDLSCs cultured in osteogenic induction conditions for 3 weeks, mineralized nodules were detected by Alizarin Red staining; magnification, x100.

A

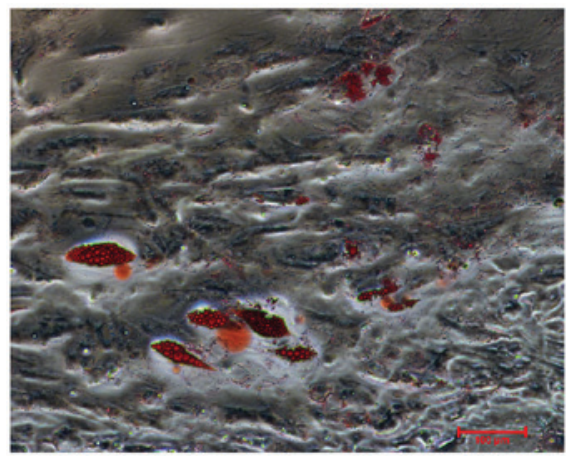

B

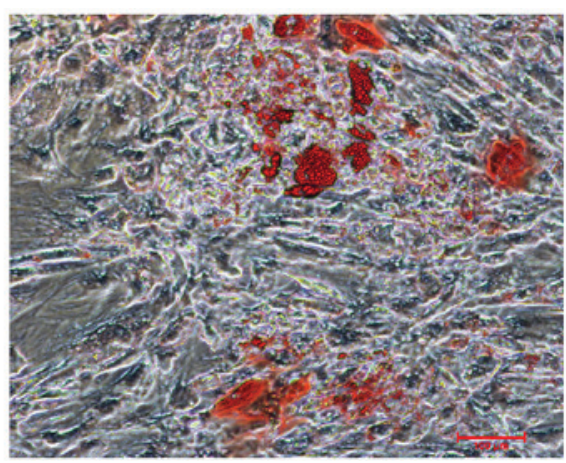

Figure 3. Adipogenic differentation of (A) gingival-derived mesenchymal stem cells (GMSCs), and (B) periodontal ligament stem cells (PDLSCs); magnification, x100. Cultured GMSCs and PDLSCs formed lipid clusters that were positively stained for Oil Red O following 3 weeks of adipogenic differentiation.

indicate a statistically significant difference. All procedures were performed blinded.

\section{Results}

Characterization of PDLSCs and GMSCs. Using the limiting dilution technique, putative clonogenic GMSCs and PDLSCs were isolated and purified. They were identified by the positive expression of STRO-1 and CD105, and negative expression of CD45 (Fig. 1). To investigate the differentiation potential of GMSCs and PDLSCs, the osteogenic and adipogenic potential of the cells was investigated. Osteogenesis was confirmed by the presence of red-stained mineral nodules (Fig. 2), and adipogenesis was confirmed by intracellular lipid droplet accumulation (Fig. 3).

Cell cycle analysis of GMSCs and PDLSCs co-cultured with $A P T G-C M$. Cell cycle analysis was performed to investigate whether APTG-CM was able to affect cell proliferation in vitro. In GMSCs and PDLSCs co-cultured with APTG-CM a markedly higher percentage of cells were present in $\mathrm{S}$ and $\mathrm{G}_{2} / \mathrm{M}$ phases (GMSCs, 35.1\%; PDLSCs, 26.3\%) whereas a lower percentage of cells were present in $\mathrm{G}_{0} / \mathrm{G}_{1}$ phase (Fig. 4) compared with in the untreated cells. These results suggest that APTG-CM may exert a stimulating effect on GMSCs and PDLSCs proliferation.

ALP activity assay. ALP is an early marker of dental MSC differentiation towards the cementoblastic/osteoblastic phenotype (25). In the present study, ALP activity of GMSCs and PDLSCs cultured with or without APTG-CM was measured. ALP activity was markedly higher in the co-culture groups. The effects of APTG-CM on GMSCs and PDLSCs increased in a time-dependent manner, up to day 12 , during the 15 -day culture period. As presented in Fig. $5(\mathrm{P}<0.05)$, ALP activity had steadily increased in the co-culture group by day 3 , and 

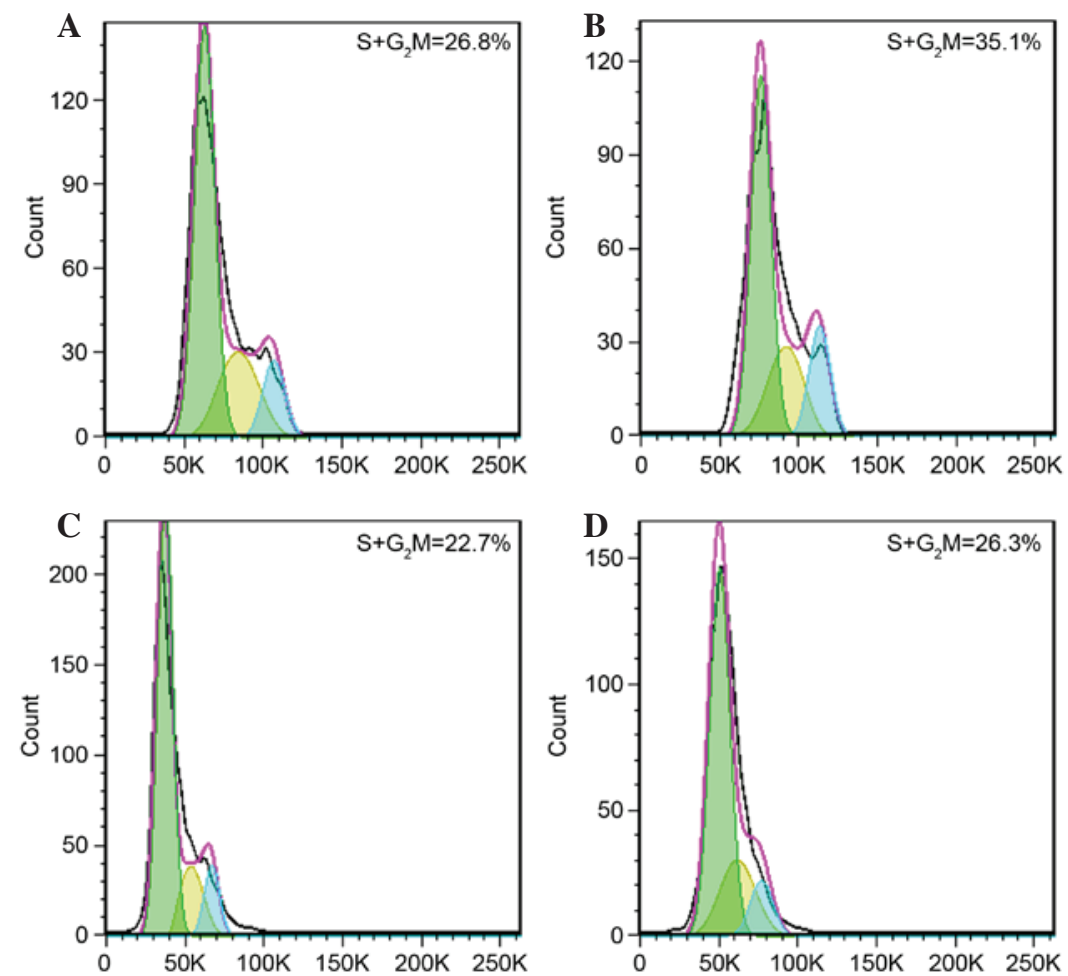

Figure 4. Cell cycle analysis of (A and B) gingival-derived mesenchymal stem cells (GMSCs) and (C and D) periodontal ligament stem cells (PDLSCs). (A and C) Representative cell cycle distribution of untreated GMSCs and PDLSCs. (B and D) Representative cell cycle distribution of GMSCs and PDLSCs co-cultured with apical tooth germ-conditioned medium (APTG-CM). APTG-CM-induced GMSCs and PDLSCs appeared to escape $\mathrm{G}_{0} / \mathrm{G}_{1}$ arrest and progressed to $\mathrm{S}$ and $\mathrm{G}_{2} / \mathrm{M}$ phases.
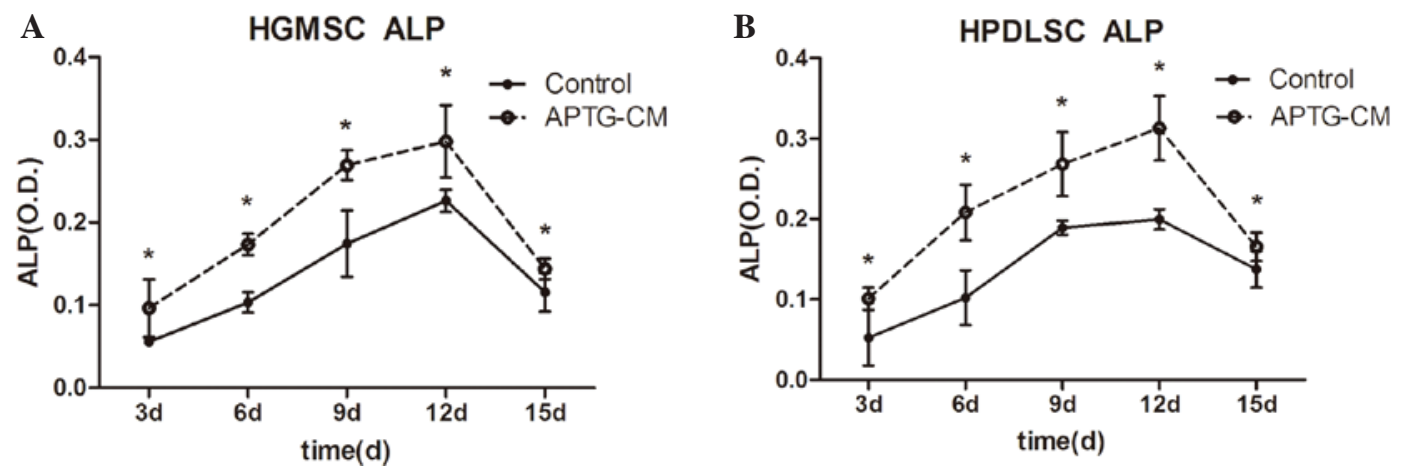

Figure 5. Alkaline phosphatase (ALP) activity of (A) gingival-derived mesenchymal stem cells (GMSCs) and (B) periodontal ligament stem cells (PDLSCs) co-cultured with apical tooth germ-conditioned medium (APTG-CM). ALP activity was increased in the APTG-CM groups compared with in the control group. ${ }^{\mathrm{P}}<0.05$. O.D, optical density.

continued to increase until it reached a peak at day 12 , thus indicating that co-cultured cells continually differentiate into hard tissue-forming cells. After day 12, ALP activity gradually decreased until day 15 , which may be correlated with increasing mineral deposition.

Gene expression in GMSCs and PDLSCs co-cultured with $A P T G-C M$. RT-PCR analysis was conducted following co-culture of the two cell types with APTG-CM. The expression levels of bone- and extracellular matrix-associated genes (Table I) were upregulated in GMSCs (Fig. 6A) and PDLSCs (Fig. 6B) co-cultured with APTG-CM compared with cells in the control group $(\mathrm{P}<0.05)$. The mRNA expression patterns of bone-related genes suggest that GMSCs and PDLSCs co-cultured with APTG-CM exhibit some molecular properties of cementoblast-like cells.

Differentiation of GMSCs and PDLSCs following heterotopic transplantation. A total of 30 complexes were harvested after 8 weeks of transplantation and evaluated by light microscopy following $\mathrm{H} \& \mathrm{E}$ staining (Fig. 7). In the experimental group (APTG-CM GMSC sheet + dentin + CBB), a cementum/PDL-like structure was regenerated on dentin surfaces, and the collagen content and orientation of PDL-like tissues were markedly obvious (Fig. 7B and C); similar characteristics were observed in the positive control group (APTG-CM PDLSC sheet + dentin + CBB; Fig. 7D and E). Furthermore, distinctive dental cementum-like matrices were 

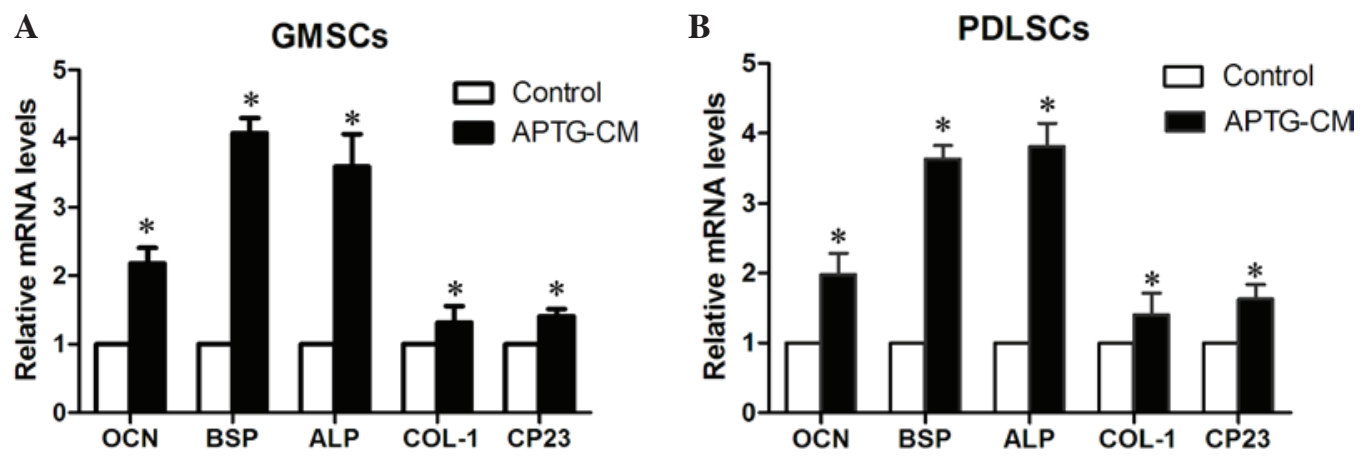

Figure 6. Bone-associated and extracellular matrix-associated gene expression was detected using reverse transcription-quantitative polymerase chain reaction before and after co-culturing gingival-derived mesenchymal stem cells (GMSCs) and periodontal ligament stem cells (PDLSCs) with apical tooth germ-conditioned medium (APTG-CM). The results revealed that (A) GMSCs and (B) PDLSCs exhibited upregulated osteocalcin (OCN), bone sialoprotein (BSP), alkaline phosphatase (ALP), type 1 collagen (COL-1) and cementum-derived protein 23 (CP23) expression following APTG-CM co-culture (n=9 each). ${ }^{*} \mathrm{P}<0.05$ vs. the control.
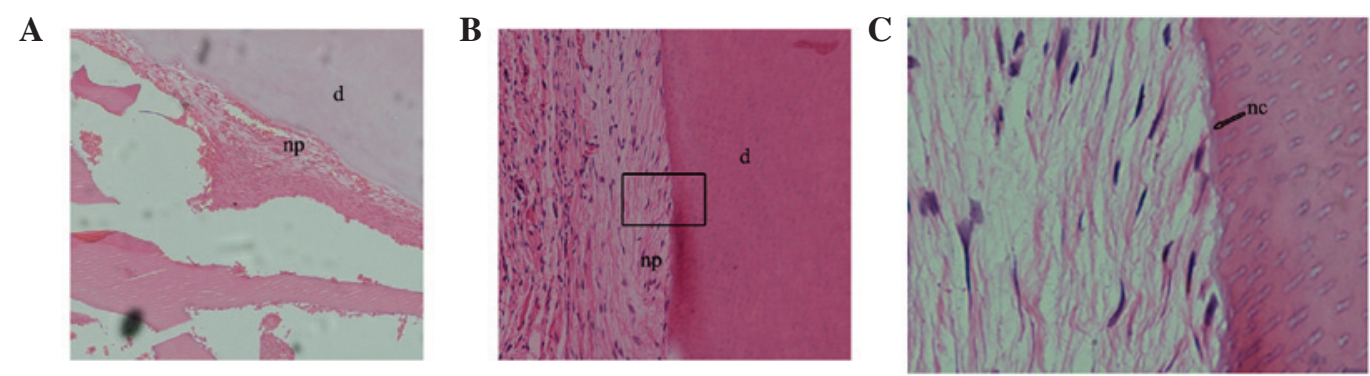

D

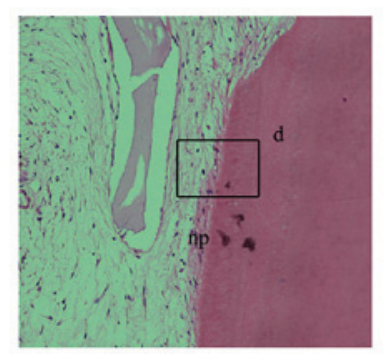

E

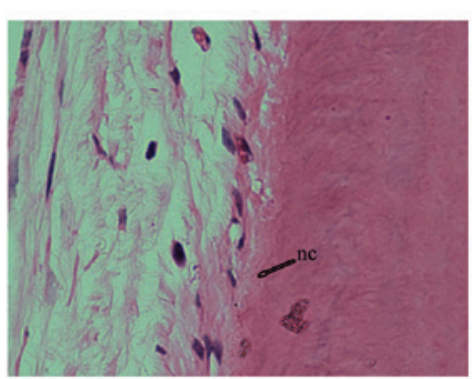

Figure 7. Following subcutaneous transplantation for 8 weeks, the differentiation potential of gingival-derived mesenchymal stem cells (GMSCs) and periodontal ligament stem cells (PDLSCs) co-cultured with apical tooth germ-conditioned medium (APTG-CM) were determined in vivo after hematoxylin and eosin staining. (A) Non-co-cultured GMSCs formed a small amount of periodontal ligament (PDL)-like tissue but no cementum-like deposits; magnification, x100. (B) GMSCs co-cultured with APTG-CM exhibited tissue-regenerative capacity, were able to produce cementum-like mineralized deposits on the surface of dentin slices, and PDL-like collagen fibers connected with the newly formed cementum-like tissues; magnification, $\mathrm{x} 100 \mathrm{x}$. (C) Magnification of the rectangular area in (B) (magnification, x400). (D) PDLSCs co-cultured with APTG-CM group also formed cementum and PDL-like structures; magnification, x100. (E) Magnification of the rectangular area in (D) (magnification, x400). np, new PDL-like collagen fibers; nc: new cementum-like tissues; d, dentin.

regenerated and clearly observed on dentin after transplantation for 8 weeks. Conversely, non-co-cultured GMSC sheet transplants (GMSC sheet + dentin + CBB) formed small amounts of PDL-like tissue but no cementum-like deposits (Fig. 7A). The images were captured on a compound microscope.

\section{Discussion}

Ideal periodontal reconstruction would involve the development of Sharpey's fibers, which consist of collagen fibers. Therefore, generation of a suitable regenerative periodontal environment is of great importance. Furthermore, a mineralized matrix is essential for periodontal regeneration. Certain osteoinductive technologies, including the use of conditioned medium from developing apical tooth germ cells, periapical follicle stem cells and Hertwig's epithelial root sheath cells, as well as osteoinductive medium, have previously been applied to create a regenerative microenvironment (4,26-29). The present study developed a periodontal complex using APTG-CM-induced GMSC sheets, dentin slices and CBB for periodontal regeneration. As hypothesized, transplantation of immunodeficient mice with this periodontal complex resulted in the generation of a dental cementum/PDL-like complex. These results indicated that the development of this periodontal complex may provide an alternative clinical approach for tooth reconstruction in future therapeutic strategies.

In the present study, the APTG-CM used may contain several molecular signals and growth factors that are necessary for GMSC and PDLSC proliferation and differentiation, 
thus inducing differentiation of GMSCs and PDLSCs towards a cementoblast phenotype. As expected, the induced GMSCs exhibited several crucial characteristics of cementoblast-like cells. Firstly, flow cytometric cell cycle analysis demonstrated that cells co-cultured with APTG-CM presented a higher percentage of cells in $S$ and $\mathrm{G}_{2} / \mathrm{M}$ phases. These results suggested that DNA synthesis was enhanced and APTG-CM may provide an appropriate microenvironment, which is necessary for the proliferation and differentiation of GMSCs and PDLSCs. Secondly, ALP activity of GMSCs and PDLSCs co-cultured with APTG-CM was increased. It is well-known that when odontogenic mesenchymal cells are differentiated towards cementum-like and osteogenic phenotypes, ALP activity is an early marker (30). The increase in ALP activity indicated that the mineralization ability of GMSCs and PDLSCs co-cultured with APTG-CM was enhanced. ALP is considered to be a prerequisite, which has a major role in the formation of mineral tissue. Furthermore, several important bone-associated genes, including osteocalcin, bone sialoprotein, ALP, type I collagen and cementum-derived protein 23 were upregulated in GMSCs and PDLSCs co-cultured with APG-CM, which may enhance PDL-like tissue regeneration. These bone-associated genes were also important markers associated with mineral extracellular matrix (31). These changes may be considered the mechanistic basis for altering the fates of GMSCs and PDLSCs, and may contribute to the regeneration of periodontal tissue. The results of an in vivo study detected similar changes in GMSCs and PDLSCs induced by APTG-CM.

The results of the in vivo heterotopic transplantation were consistent with the results of the in vitro study. In the experimental and positive control groups, PDL-like structures were regenerated on the dentin surfaces and novel cementum matrix generation was detected. These findings were the most important in the present study; to the best of our knowledge, there are no reports regarding the use of GMSCs to replace PDLSCs in periodontal regeneration, and the subsequent regeneration of a PDL-like structure. However, it is worth noting that the formation of PDL-like structures was continuous, this may be due to the stability and compactness between cell sheets and dentin slices due to the surgical sutures used, and may be associated with novel cementum matrix generation, it is well-known that acellular cementum regeneration is the gold standard of periodontal regeneration (32). Still, it was related to the environmental factors where the complex located. In the present study, only heterotopic transplantation was used to simulate periodontal regeneration. A previous study demonstrated that the alveolar bone environment differs from that of other areas (33) and is suitable for periodontal regeneration (34). If the complexes were orthotopic transplants into jaws, the microenvironment would be better suited to periodontal regeneration, this could be a target of future investigation. Angiogenesis may also be important. In the present study regeneration of the PDL-like structure was accompanied by angiogenesis. Restoration of vascular supply was considered to be a critical factor in successful tissue engineering $(35,36)$. Additional studies are required to confirm the differences between periodontal tissue formed by GMSCs and PDLSCs.

In conclusion, the results of the present study suggested that GMSCs induced by APTG-CM have the potential for periodontal tissue regeneration. This may have general implications for periodontal engineering. Future research is required to confirm the differences between periodontal tissue formed by GMSCs and PDLSCs.

\section{Acknowledgements}

The present study was supported by the National Natural Science Foundation of China (grant no. 81271152).

\section{References}

1. Chen FM, Zhang J, Zhang M, An Y, Chen F and Wu ZF: A review on endogenous regenerative technology in periodontal regenerative medicine. Biomaterials 31: 7892-7927, 2010.

2. Chen FM and Jin Y: Periodontal tissue engineering and regeneration: Current approaches and expanding opportunities. Tissue Eng Part B Rev 16: 219-255, 2010.

3. Seo BM, Miura M, Gronthos S, Bartold PM, Batouli S, Brahim J, Young M, Robey PG, Wang CY and Shi S: Investigation of multipotent postnatal stem cells from human periodontal ligament. Lancet 364: 149-155, 2004.

4. Naveau A, Reinald N, Fournier B, Durand E, Lafont A, Coulomb B and Gogly B: Gingival fibroblasts inhibit MMP-1 and MMP-3 activities in an ex-vivo artery model. Connect Tissue Res 48: 300-308, 2007.

5. Zhang Q, Shi S, LiuY, Uyanne J, Shi Y, Shi S and Le AD: Mesenchymal stem cells derived from human gingiva are capable of immunomodulatory functions and ameliorate inflammation-related tissue destruction in experimental colitis. J Immunol 183: 7787-7798, 2009.

6. Mitrano TI, Grob MS, Carrión F, Nova-Lamperti E, Luz PA, Fierro FS, Quintero A, Chaparro A and Sanz A: Culture and Characterization of mesenchymal stem cells from human gingival tissue. J Periodontol 81: 917-925, 2010

7. Wang F, Yu M, Yan X, Wen Y, Zeng Q, Yue W, Yang P and Pei X: Gingival-derived mesenchymal stem cell-mediated therapeutic for bone tissue regeneration. Stem Cells Dev 20: 2093-2102, 2011.

8. Zhang QZ, Su WR, Shi SH, Wilder-Smith P, Xiang AP, Wong A Nguyen AL, Kwon CW and Le AD: Human gingiva-derived mesenchymal stem cells elicit polarization of M2 macrophages and enhance cutaneous wound healing. Stem Cells 28: 1856-1868, 2010.

9. Kushida A, Yamato M, Konno C, Kikuchi A, Sakurai Y and Okano T: Decrease in culture temperature releases monolayer endothelial cell sheets together with deposited fibronectin matrix from temperature-responsive culture surfaces. J Biomed Mater Res 45: 355-362, 1999.

10. Yang J, Yamato M, Kohno C, Nishimoto A, Sekine H, Fukai F and Okano T: Cell sheet engineering: Recreating tissues without biodegradable scaffolds. Biomaterials 26: 6415-6422, 2005.

11. Hasegawa M, Yamato M, Kikuchi A, Okano T and Ishikawa I: Human periodontal ligament cell sheets can regenerate periodontal ligament tissue in an athymic rat model. Tissue Eng 11: 469-478, 2005

12. Yang Z, Jin F, Zhang X, Ma D, Han C, Huo N, Wang Y, Zhang Y, Lin Z and Jin Y: Tissue engineering of cementum/periodontal-ligament complex using a novel three-dimensional pellet cultivation system for human periodontal ligament stem cells. Tissue Eng Part C Methods 15: 571-581, 2009.

13. Iwata T, Yamato M, Tsuchioka H, Takagi R, Mukobata S, Washio K, Okano T and Ishikawa I: Periodontal regeneration with multi-layered periodontal ligament derived cell sheets in a canine model. Biomaterials 30: 2716-2723, 2009.

14. Koyanagi M, Brandes RP, Haendeler J, Zeiher AM and Dimmeler S: Cell-to-cell connection of endothelial progenitor cells with cardiac myocytes by nanotubes: a novel mechanism for cell fate changes? Circ Res 96: 1039-1041, 2005.

15. Kikuchi H, Suzuki K, Sakai N and Yamada S: Odontoblasts induced from mesenchymal cells of murine dental papillae in three-dimensional cell culture. Cell Tissue Res 317: 173-185, 2004.

16. Smith AJ and Lesot H: Induction and regulation of crown dentinogenesis: Embryonic events as a template for dental tissue repair? Crit Rev Oral Biol Med 12: 425-437, 2001. 
17. Yu JH, Shi JN, Deng ZH, Zhuang H, Nie X, Wang RN, and Jin Y: Cell pellets from dental papillae can reexhibit dental morphogenesis and dentinogenesis. Biochem Biophys Res Commun 346: 116-124, 2006.

18. Harada H, Toyono T, Toyoshima K, Yamasaki M, Itoh N, Kato S, Sekine K and Ohuchi H: FGF10 maintains stem cell compartment in developing mouse incisors. Development 129: 1533-1541, 2002.

19. Tummers M and Thesleff I: Root or crown: A developmental choice orchestrated by the differential regulation of the epithelial stem cell niche in the tooth of two rodent species. Development 130 1049-1057, 2003.

20. Harada $\mathrm{H}$ and Ohshima $\mathrm{H}$ : New perspectives on tooth development and the dental stem cell niche. Arch Histol Cytol 67: 1-11, 2004.

21. Nakashima M: Bone morphogenetic proteins in dentin regeneration for potential use in endodontic therapy. Cytokine Growth Factor Rev 16: 369-376, 2005

22. Ohshima H, Nakasone N, Hashimoto E, Sakai H, Nakakura-Ohshima $\mathrm{K}$ and Harada $\mathrm{H}$ : The eternal tooth germ is formed at the apical end of continuously growing teeth. Arch Oral Biol 50: 153-157, 2005.

23. Livak KJ and Schmittgen TD: Analysis of relative gene expression data using real-time quantitative PCR and the 2(-Delta Delta C(T)) method. Methods 25: 402-408, 2001.

24. Wittekind D: Traditional staining for routine diagnostic pathology including the role of tannic acid. 1. Value and limitations of the hematoxylin-eosin stain. Biotech Histochem 78: 261-270, 2003.

25. Thomas HF and Kollar EJ: Differentiation of odontoblasts in grafted recombinants of murine epithelial root sheath and dental mesenchyme. Arch Oral Biol 34: 27-35, 1989.

26. Han C, Yang Z, Zhou W, Jin F, Song Y, Wang Y, Huo N, Chen L, Qian H, Hou R, et al: Periapical follicle stem cell: A promising candidate for cementum/periodontal ligament regeneration and bio-root engineering. Stem Cells Dev 19: 1405-1415, 2009.

27. Flores MG, Hasegawa M, Yamato M, Takagi R, Okano $\mathrm{T}$ and Ishikawa I: Cementum-periodontal ligament complex regeneration using the cell sheet technique. J Periodontal Res 43: 364-371, 2008.
28. Flores MG, Yashiro R, Washio K, Yamato M, Okano T and Ishikawa I: Periodontal ligament cell sheet promotes periodontal regeneration in athymic rats. J Clin Periodontol 35: 1066-1072, 2008.

29. Bai Y, Bai Y, Matsuzaka K, Hashimoto S, Fukuyama T, Wu L, Miwa T, Liu X, Wang X and Inoue T: Cementum- and periodontal ligament-like tissue formation by dental follicle cell sheets co-cultured with Hertwig's epithelial root sheath cells. Bone 48: 1417-1426, 2011.

30. Sumita Y, Honda MJ, Ohara T, Tsuchiya S, Sagara H, Kagami $\mathrm{H}$ and Ueda M: Performance of collagen sponge as a 3-D scaffold for tooth-tissue engineering. Biomaterials 27: 3238-3248, 2006.

31. Kumar S, Mahendra G and Ponnazhagan S: Determination of osteoprogenitor-specific promoter activity in mouse mesenchymal stem cells by recombinant adeno-associated virus transduction. Biochim Biophys Acta 1731: 95-103, 2005.

32. Grzesik WJ and Narayanan AS: Cementum and periodontal wound healing and regeneration. Crit Rev Oral Biol Med 13: 474-484, 2002.

33. Leucht P, Kim JB, Amasha R, James AW, Girod S and Helms JA: Embryonic origin and hox status determine progenitor cell fate during adult bone regeneration. Development 135: 2845-285414, 2008.

34. Ikeda E, Morita R, Nakao K, Ishida K, Nakamura T, Takano-Yamamoto T, Ogawa M, Mizuno M, Kasugai S and Tsuji T: Fully functional bioengineered tooth replacement as an organ replacement therapy. Proc Natl Acad Sci USA 106: 13475-13480, 2009.

35. Kim BS and Mooney DJ: Development of biocompatible synthetic extracellular matrices for tissue engineering. Trends Biotechnol 16: 224-230, 1998.

36. Polykandriotis E, Arkudas A, Horch RE, Stürzl M and Kneser U: Autonomously vascularized cellular constructsin tissue engineering: Opening a new perspective for biomedical science. J Cell Mol Med 11: 6-2014, 2007. 